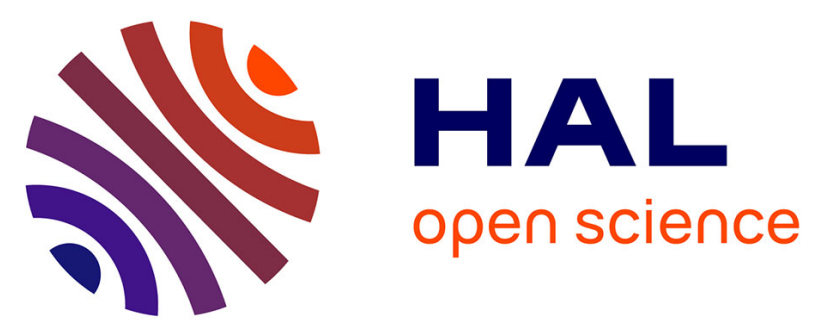

\title{
Advanced In Vivo Cross-Linking Mass Spectrometry Platform to Characterize Proteome-Wide Protein Interactions
}

\author{
Martial Jean-Pierre Rey, Jonathan Dhenin, Youxin Kong, Lucienne
} Nouchikian, Isaac Filella, Magalie Duchateau, Mathieu Dupré, Riccardo Pellarin, Guillaume Duménil, Julia Chamot-Rooke

\section{To cite this version:}

Martial Jean-Pierre Rey, Jonathan Dhenin, Youxin Kong, Lucienne Nouchikian, Isaac Filella, et al.. Advanced In Vivo Cross-Linking Mass Spectrometry Platform to Characterize Proteome-Wide Protein Interactions. Analytical Chemistry, 2021, 93 (9), pp.4166-4174. 10.1021/acs.analchem.0c04430 . hal03157343

\section{HAL Id: hal-03157343 \\ https://hal.science/hal-03157343}

Submitted on 14 Oct 2021

HAL is a multi-disciplinary open access archive for the deposit and dissemination of scientific research documents, whether they are published or not. The documents may come from teaching and research institutions in France or abroad, or from public or private research centers.
L'archive ouverte pluridisciplinaire $\mathbf{H A L}$, est destinée au dépôt et à la diffusion de documents scientifiques de niveau recherche, publiés ou non, émanant des établissements d'enseignement et de recherche français ou étrangers, des laboratoires publics ou privés.

\section{(ㅇ)(1) $\$$}

Distributed under a Creative Commons Attribution - NonCommercial - NoDerivatives| 4.0 


\title{
Advanced In Vivo Cross-Linking Mass Spectrometry Platform to Characterize Proteome-Wide Protein Interactions
}

\author{
Martial Rey,* Jonathan Dhenin, Youxin Kong, Lucienne Nouchikian, Isaac Filella, Magalie Duchateau, \\ Mathieu Dupré, Riccardo Pellarin, Guillaume Duménil, and Julia Chamot-Rooke*
}

Cite This: Anal. Chem. 2021, 93, 4166-4174

Read Online

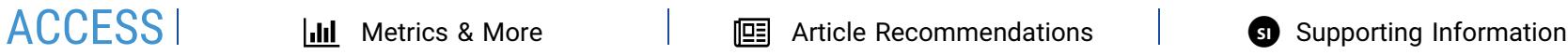

ABSTRACT: Chemical cross-linking (XL) coupled to mass spectrometry (MS) has become a powerful approach to probe the structure of protein assemblies. Although most of the applications concerned purified complexes, latest developments focus on large-scale in vivo studies. Pushing in this direction, we
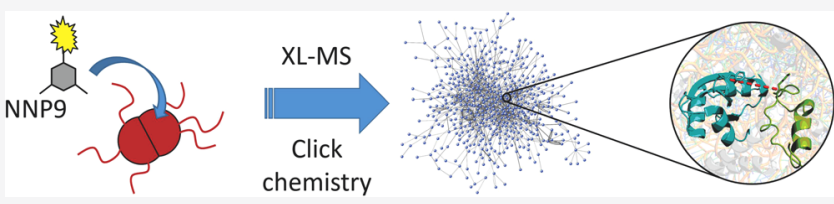
developed an advanced in vivo cross-linking mass spectrometry platform to study the cellular interactome of living bacterial cells. It is based on in vivo labeling and involves a one-step enrichment by click chemistry on a solid support. Our approach shows an impressive efficiency on Neisseria meningitidis, leading to the identification of about 3300 cross-links for the LC-MS/MS analysis of a biological triplicate using a benchtop high-resolution Orbitrap mass spectrometer. Highly dynamic multiprotein complexes were successfully captured and characterized in all bacterial compartments, showing the great potential and precision of our proteome-wide approach. Our workflow paves new avenues for the large-scale and nonbiased analysis of protein-protein interactions. All raw data, databases, and processing parameters are available on ProteomeXchange via PRIDE repository (data set identifier PXD021553).

\section{INTRODUCTION}

In past years, chemical cross-linking (XL) combined with mass spectrometry (MS) has become an approach of choice to probe the structures of proteins and protein complexes. Although most of the applications concerned purified protein complexes, latest developments focus on large-scale ${ }^{1,2}$ and in vivo $^{3-6}$ experiments for proteome-wide studies. This step forward was made possible thanks to improvements at multiple steps of the workflow: (i) cross-linking of the sample, (ii) enrichment of the cross-linked peptides, (iii) nano-liquid chromatography coupled to mass spectrometry (nanoLCMSn) analysis of the purified labeled peptides, and (iv) data processing using dedicated high-end search engines. Nevertheless, there are still limitations that cause in vivo XL-MS to be nowhere near the speed, the sensitivity, and the depth of a regular proteomics experiment.

The first and main challenge comes with the low abundance of the cross-linked peptides after the proteolysis step and the complexity of the peptide pool generated. Indeed, if proper experimental conditions are used to avoid unspecific intermolecular interactions, only a few cross-linked proteins (and thus peptides) are generated, which largely complicates their identification especially for in vivo studies. To face this issue, various enrichment strategies were developed like strong cation exchange $(\mathrm{SCX})^{7-11}$ or size exclusion chromatography (SEC), ${ }^{12,13}$ alone or in combination, both requiring a significant amount of starting material. ${ }^{3}$ For example, Ryl et al. combined multiple fractionation steps with sequential digestion (trypsin followed by various proteases) to study the mitochondrial interactome. Combining a total of 110 LC-MS/ MS runs, they identified 5518 unique residue pairs from 792 proteins. ${ }^{3}$ This result illustrates the huge gap remaining with regular proteomics analysis, where tens of thousands of peptides and a few thousands of proteins can be routinely identified in a single LC-MS/MS run. ${ }^{14}$

To be efficient, the purification strategy required for XL-MS studies needs to combine (i) a high specificity to capture all modified peptides including those of very low abundance, (ii) a high affinity to allow extensive washing to remove all nonlabeled peptides without loss of the labeled material, and finally (iii) efficient release conditions. To this aim, enrichment strategies employing biotinylated cross-linkers and trapping on avidin beads were developed. ${ }^{15,16}$ The latest improvement was to use reagents carrying either an alkyne or an azido group, allowing the biotin tag to be introduced by click chemistry in a second step since cross-linking reagents with bulky affinity tags may impair the labeling reaction. ${ }^{17-19}$ Using affinity-based enrichment, Sohn et al. successfully identified cross-linked ubiquitin peptides diluted in yeast lysate, thus demonstrating the benefit of an affinity-based enrichment. ${ }^{18}$ However, the

Received: October 20, 2020

Accepted: February 5, 2021

Published: February 22, 2021 
high affinity of biotin to streptavidin prevents the efficient release of the labeled peptides.

Another challenge is the high complexity of the peptide mixture obtained after the enzymatic digestion. Indeed, a mixture of unmodified, singly modified (called dead-end or monolink), and both intra- and intermolecular cross-linked peptides is generated. A strategy that has been developed to ease the identification of cross-linked peptides is the use of isotopically labeled cross-linkers that give rise to a specific signature in MS spectra, allowing their specific fragmentation. $^{20-22}$ Moreover, at the MS2 level, a comparison between light and heavy forms allows precise localization of the crosslinker site: the fragments presenting the same mass in the light and heavy forms are not labeled, while the fragments containing the cross-linker present specific mass shifts. ${ }^{7}$ Although this method is quite appealing, the signal intensity of the cross-linked peptides is split into (and thus divided by) two, making the lowest-abundance peaks disappear in the background noise. The use of isotopically labeled cross-linkers also increases spectral complexity, which is prohibitive for in vivo studies.

Finally, the last challenge in the development of efficient in vivo XL-MS workflows is data processing. The major issue comes from the fact that comparing large experimental data sets to all potential peptide pair combinations increases the search space exponentially in regard to the number of peptides. This well-described $n^{2}$ times increase of the complexity to search for cross-linked peptides becomes particularly acute when searching against a whole proteome. ${ }^{7,23}$ In past years, many dedicated software solutions have been released using different strategies to automatically identify cross-linked peptides from LC-MS ${ }^{n}$ experiments, some with statistical analysis and graphical outputs. ${ }^{7,24-28} \mathrm{New}$ cross-linkers that are cleavable in the gas phase through collision-induced dissociation (CID) or electron transfer dissociation (ETD) have also emerged to reduce the time complexity of the search. ${ }^{6,29-31}$ This approach consists in releasing each part of a cross-linked peptide in an $\mathrm{MS}^{2}$ experiment and treating each $\mathrm{MS}^{3}$ experiment as for a linear peptide identification. Despite its efficiency to reduce the space search, this approach generates two pairs of fragments in $\mathrm{MS}^{2}$ that need to be fragmented in $\mathrm{MS}^{3}$. In total, one $\mathrm{MS}^{2}$ and four $\mathrm{MS}^{3}$ are therefore required to identify one cross-linked peptide. ${ }^{32}$ This increase in the cycle time automatically limits the number of potential cross-linked peptides that can be analyzed, thus impairing the depth of the analysis.

With the aim of implementing an efficient and reliable XLMS method applicable to a wide range of biological systems, our group has developed, a few years ago, a trifunctional crosslinking agent, named "NNP9." 33 It bears NHS-carbamate groups, which are more stable in aqueous solution than the classical NHS-esters, and thus improves the cross-linking efficiency. NNP9 also carries an azido "clickable" group that allows further enrichment of cross-linked peptides. ${ }^{19,33}$

We propose here a new purification approach, based on the use of NNP9, designed to capture all labeled peptides with a minimal loss of material and a simple and very efficient releasing step in an MS-friendly buffer. We also took advantage of the latest software capable of identifying cross-linked peptides directly from $\mathrm{MS}^{2}$ data. ${ }^{28}$ Thanks to this new XLMS pipeline, we studied the interactome of a bacterial pathogen, Neisseria meningitidis. It is of high interest to characterize protein complexes or protein networks involved in the virulence of this human pathogen or important for its survival as they can be potential targets for vaccine development. For instance, one current strategy for vaccine development against Neisseria is to target the bacterial iron import systems that are critical for its survival and well conserved among bacterial species. ${ }^{34}$ Applied on N. meningitidis living cells, the results obtained show an impressive efficiency with about 3300 cross-links identified for the LC-MS/MS analysis of a biological triplicate on an Orbitrap benchtop instrument. Moreover, we were able to capture structural details of dynamic protein complexes in their functional state present in all compartments of the meningococcus, from the cytoplasm to the outer membrane, highlighting the great interest of such in vivo studies.

\section{EXPERIMENTAL SECTION}

Products. NNP9 was synthetized by the COBRA lab in Rouen and stored dissolved in DMSO at $100 \mathrm{mM}$ (Figure S1). Phosphate buffer saline, ammonium bicarbonate, copper(II) sulfate, Tris(3-hydroxypropyltriazolylmethyl)amine (THPTA), and sodium ascorbate were purchased from Sigma-Aldrich. Photocleavable alkyne agarose beads were purchased from Jena Bioscience. A UV lamp (100 W) was purchased from Analytik Jena US, and 96-well plates and Amicon Ultra $(0.5 \mathrm{~mL}, 30$ $\mathrm{kDa})$ were purchased from Dominique Dutscher.

N. meningitidis Cross-Linking. $N$. meningitidis ( $\mathrm{Nm} 8013)$ cells were grown overnight on a GBC plate supplemented with ferric citrate. Cells were harvested and washed three times with PBS. Then, $10 \mu \mathrm{L}$ of cells was diluted 10 times with PBS, and 2 $\mu \mathrm{L}$ of NNP9 $(100 \mathrm{mM}, 200 \mathrm{nmol})$ was added. After $1 \mathrm{~h}$ at 37 ${ }^{\circ} \mathrm{C}, 200 \mathrm{nmol}$ of NNP9 was added again with 1\% DDM (this step was repeated twice). The cross-linking reaction was stopped by addition of ammonium bicarbonate $(100 \mathrm{mM}$ final concentration) on ice for $15 \mathrm{~min}$. Cells were lysed with $6 \mathrm{M}$ urea, final concentration.

Protein Digestion. Cross-linked proteins were transferred into passivated Amicon Ultra $(0.5 \mathrm{~mL}, 30 \mathrm{kDa})$. Excess of cross-linker was removed by six concentration-dilution cycles with $50 \mathrm{mM}$ ammonium bicarbonate. Proteins were digested overnight with a trypsin ratio of 1:100 (w/w). Tryptic peptides were recovered through the filter after centrifugation.

Enrichment of Labeled Peptides. Photocleavable alkyne agarose beads (PCABs) $(1 \mu \mathrm{L}: 10 \mathrm{nmol})$ were mixed with the tryptic peptides, copper(II) sulfate (5:1), THPTA (25:1), and sodium ascorbate $(50: 1)$, where the ratios are given in regard to NNP9 quantity. After $30 \mathrm{~min}$ of side rotation at room temperature, PCABs were washed four times with PBS and twice with $0.1 \%$ formic acid to remove unlabeled peptides. PCABs were transferred to a 96-well plate (maximum $10 \mu \mathrm{L}$ of PCABs per well), covered with $50 \mu \mathrm{L}$ of $0.1 \%$ formic acid, and placed under UV for $10 \mathrm{~min}$ followed by rotational mixing $(500 \mathrm{rpm})$. Released peptides were pipetted and transferred into a polypropylene injection vial.

N. meningitidis Whole Proteome Analysis. N. meningitidis cells were solubilized in urea, $8 \mathrm{M}$, and Tris, $100 \mathrm{mM}$, $\mathrm{pH}$ 7.5; then, disulfide bonds were reduced with $5 \mathrm{mM}$ tris(2carboxyethyl)phosphine (TCEP) for $20 \mathrm{~min}$ at $23{ }^{\circ} \mathrm{C}$ and alkylated with $20 \mathrm{mM}$ iodoacetamide in the dark for $30 \mathrm{~min}$ at room temperature. Subsequently, LysC was added for the first digestion step (protein-to-LysC ratio $=80: 1$ ) for $3 \mathrm{~h}$ at $30{ }^{\circ} \mathrm{C}$. Then, the samples were diluted to $1 \mathrm{M}$ urea with $100 \mathrm{mM}$ Tris, $\mathrm{pH} 7.5$, and sequencing-grade modified trypsin was added to the sample at a ratio of $50: 1$ for $16 \mathrm{~h}$ at $37^{\circ} \mathrm{C}$. Proteolysis was 


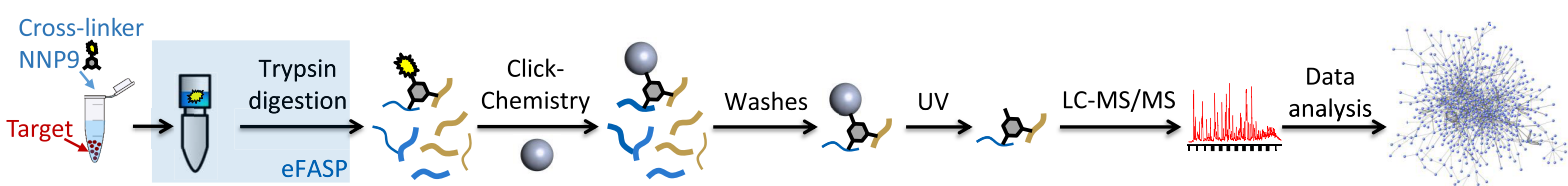

Figure 1. Pipeline for in vivo cross-linking mass spectrometry. Bacteria or purified protein complexes are mixed with a cross-linker, NNP9. Filteraided sample preparation (eFASP) is performed to wash away the excess of cross-linker. Proteins are then digested overnight with trypsin. Modified peptides are purified using click chemistry on photocleavable beads, released through photodissociation after extensive washes, and analyzed by LCMS/MS. Data are processed using Mass Spec Studio.

stopped by adding $1 \%$ formic acid. Resulting peptides were desalted using a Sep-Pak $\mathrm{C}_{18}$ SPE cartridge according to manufacturer's instructions.

N. meningitidis Whole Proteome MS Analysis. The bottom-up proteomics analysis was performed as described in Dupré et al. 2020. ${ }^{35}$

N. meningitidis Peptides' MS Analysis. Peptides eluted from PCABs were analyzed by nanoLC-MS/MS using an EASY-nLC 1200 system (Thermo-Scientific) coupled to the nanoelectrospray ion source of an Orbitrap Q-Exactive HF mass spectrometer (Thermo-Scientific). Peptides were loaded on an in-house packed nano-HPLC column $(75 \mu \mathrm{m} \times 50 \mathrm{~cm})$ with $\mathrm{C}_{18}$ resin (Aeris PEPTIDE XB-C18, $1.7 \mu \mathrm{m}$ particles, 100 $\AA$ pore size, Phenomenex) and separated by reverse-phase chromatography at $250 \mathrm{~nL} / \mathrm{min}$. N. meningitidis peptides were separated using a $3 \mathrm{~h}$ linear gradient from 8 to $30 \%$ solvent $\mathrm{B}$ ( $80 \%$ acetonitrile, $0.1 \%$ formic acid) followed by a $35 \mathrm{~min}$ ramp up to $60 \%$ solvent $\mathrm{B}$. The Orbitrap mass spectrometer was set up in data-dependent acquisition mode. After a survey scan over the $m / z$ range 300-1500 in the Orbitrap (resolution $60 \mathrm{k}$ at $\mathrm{m} / z 200$ ), the 10 most intense precursor ions above 4.2 $\times 10^{5}$ intensity with 3-8 charges were selected for HCD fragmentation with a normalized collision energy (NCE) set up to 26. All raw data were processed using Mass Spec Studio V2.3. N. meningitidis data were searched using the "E-Value Generator" algorithm with Percent E Value Threshold set at 0.03 using the genome-derived protein database of our $N$. meningitidis strain NM8013, containing 2125 protein entries (see details in Supporting Information). Cross-link identifications were considered true above $95 \%$ confidence cutoff (score above 13). Identification lists were then cleaned, removing the duplicates sharing the same sequences. The cross-linked peptides with the same sequences but different cross-linking sites and different retention times were kept as 2 true different identifications. Discrepancies in the cross-linked sites were assessed manually, ensuring that two chromatographic peaks did not share the same pair of sites.

Evaluation of the Distances Captured on the Ribosome. Owing to the fact that the structure of the $N$. meningitidis ribosome is not available, we computed the distance between the cross-linked residues using the closest homologue that had the best structural information. Two species are well represented in the structural databases, namely, Escherichia coli (EC) and Thermus thermophilus (TT). To identify the closest species and associate each $N$. meningitidis subunit to a homologue subunit of EC or TT, we computed the alignment between $N$. meningitidis ribosomal protein subunits against all of the subunits of EC and TT. The criterion used to associate two subunits from different species was that their percentage identity (\% ID) was higher than $30 \%$ and that the difference in \% ID between the most similar and the second most similar subunits from the mapped species was higher than $8 \%$ (EC) or $4 \%$ (TT). We obtained that $72 \%$ (49/
68) of N. meningitidis ribosomal subunits were uniquely associated with EC with a mean \% ID of 52.27 and 64\% (44/ 68 ) of $N$. meningitidis ribosomal subunits were uniquely associated with TT with a mean \% ID of 40.23 .

To compute the distances between cross-linked lysines, we decided to use the structure of the EC ribosome bound to the elongation factor G (PDB entry number 3JA1, https:// advances.sciencemag.org/content/1/4/e1500169), which is one of the most complete structures. The domains of the ribosome subunits were represented by beads based on the available crystallographic structures and comparative models. The structures were coarse-grained by representing each consecutive residue by a bead, centered on the center of mass of the residue. Sequence segments missing in the crystal structures were substituted by multiple beads of 10 residues each, as suitable. A short coordinate optimization was run to relax the bonds of missing loops. The residue mapping on the E. coli ribosome structure was obtained through pairwise sequence alignment of each $N$. menigitidis protein against the corresponding E. coli ribosomal proteins. The positions of the cross-linked $N$. menigitidis residues were renumbered accordingly, to match E. coli lysine in the aligned position. If a $N$. menigitidis cross-linked position occurred in a gap in the E. coli sequence, the position of the closest residue in the $E$. coli sequence was considered for mapping. Pairwise alignments were performed using the Biopython library. Finally, the crosslinked distance was computed between the centers of coarsegrained residues.

The model building and the distance calculation were performed using the python modeling interface (PMI), a library to model macromolecular complexes based on our open-source IMP package (http://salilab.org/imp/), version 2.13 (Russel et al. 2012). Files containing the input data, scripts, and output models are available at https://gitlab. pasteur.fr/rpellari/ribosome_nnp9.

\section{RESULTS AND DISCUSSION}

One important improvement of our new workflow relies on an efficient purification step of the cross-linked peptides based on copper/ascorbate-catalyzed Huisgen cycloaddition (click chemistry) on an ultraviolet (UV)-cleavable support (Figure S2). ${ }^{36}$ The main difference between the current workflow and our last work ${ }^{19}$ (Rey et al. 2018) is that the enrichment of the cross-linked peptides is now done in one step by clicking the cross-linked peptides on photocleavable beads. The bioorthogonal chemical reaction creates covalent bonds, with high specificity, between the azido group of NNP9 present only in the modified peptides (cross-linked ones but also deadends) and an alkyne group present on the UV-cleavable beads (Figure S2).

After extensive washing of the nonmodified peptides, the modified ones can be released specifically in an MS-friendly buffer under UV light thanks to the UV-cleavable group 
present on the support (Figure 1). To optimize each parameter of the reaction, i.e., the ratio of the alkyne and azido groups (and thus the amount of beads) and the time and conditions of the click-chemistry reaction and of the release of modified peptides, we used an azide-containing flurorescent dye (6carboxyfluorescein, 6-FAM). It allowed us to quantify reliably the amount of clicked molecules at each step of the purification protocol (starting quantities, not bound quantities and released quantities) by simply tracking with a spectrophotometer the absorbance of the dye at $495 \mathrm{~nm}$ in solution. With optimized conditions, we succeeded in capturing more than $95 \%$ of the starting material and releasing more than $90 \%$ of the bound one, even after extensive washing of the beads after the clickchemistry reaction (Figure S3). The overall recovery was greater than $85 \%$ for the starting material quantities $(2,5$ and $10 \mathrm{nmol})$.

In Vivo Cross-Linking Analysis of $\boldsymbol{N}$. meningitidis. We first checked on myoglobin that our workflow was efficient (data not shown) and moved to the analysis of N. meningitidis. We harvested bacteria grew on a plate and quickly washed them three times with PBS. We cross-linked $10 \mu \mathrm{L}$ of the cell pellet with $0.6 \mu \mathrm{mol}$ of NNP9 for $3 \mathrm{~h}$ (with 1\% DDM for the last $2 \mathrm{~h}$ ), and after quenching, we lysed all remaining cells with $6 \mathrm{M}$ urea. We then applied all steps of our optimized protocol, from protein digestion to mass spectrometry analysis, on a QExactive HF. Data were analyzed with Mass Spec Studio, taking advantage of the database reduction strategy. ${ }^{28}$ Its smart algorithm is based on the assumption that every putative crosslinked peptide should present a modest subset of fragment ions arising from a linear segment. Using this strategy, Sarpe et al. showed that the database size could be strongly reduced without sacrificing the quality of the identification.

In a single $4 \mathrm{~h}$ run, we identified between 8871 and 9313 modified peptides per replicate belonging to 842-949 proteins, and in total 1137 proteins, which represents half the proteome of $N$. meningitidis (Table S2). Among the labeled proteins, 878 were found to be interacting with a partner. We compared our data to a whole proteome analysis performed with two different gradients of 2 and $4 \mathrm{~h}$. We respectively identify 12124 and 12834 peptides corresponding to 1413 and 1439 proteins (Table S3), which is slightly deeper than our cross-linking approach (77\% of peptides, $66 \%$ of the proteins). We also calculated that $24.5 \%$ of the MS/MS spectra lead to an identification, which is similar to a nonlabeled proteome analysis of this strain (26\% in a $4 \mathrm{~h}$ gradient and $36 \%$ in a $2 \mathrm{~h}$ gradient, Table S2). Overall, our approach is closing the gap of sensitivity to regular proteomics, especially if we consider the difficulty of identifying modified peptides, in particular, cross-linked ones compared to nonmodified peptides. Note that the protein database used for the search did not contain any modification such as N-terminal processing (methionine excision or signal peptide removal) nor specific post-translation modifications although perfectly characterized (glycosylation for instance). Searching for these modifications would result in a combinatorial explosion of possibilities, making the search impossible.

The total number of unique cross-links achieved for the biological triplicate is 3342 , representing $43 \%$ of all peptides (Table S4). This very large number is typically obtained only with extensive fractionation and not in one triplicate analysis. We split the cross-links into three categories, the inter-XLs (cross-links found between two different proteins), the intraXLs (cross-links between two peptides of the same protein), and the loop-links (LLs, two residues connected by the crosslinker but not separated by a tryptic cleavage site). We identified 878 inter-XLs, 369 intra-XLs, and 2094 LLs (Figure 2 , Table S1). The large number of LLs identified is due to two
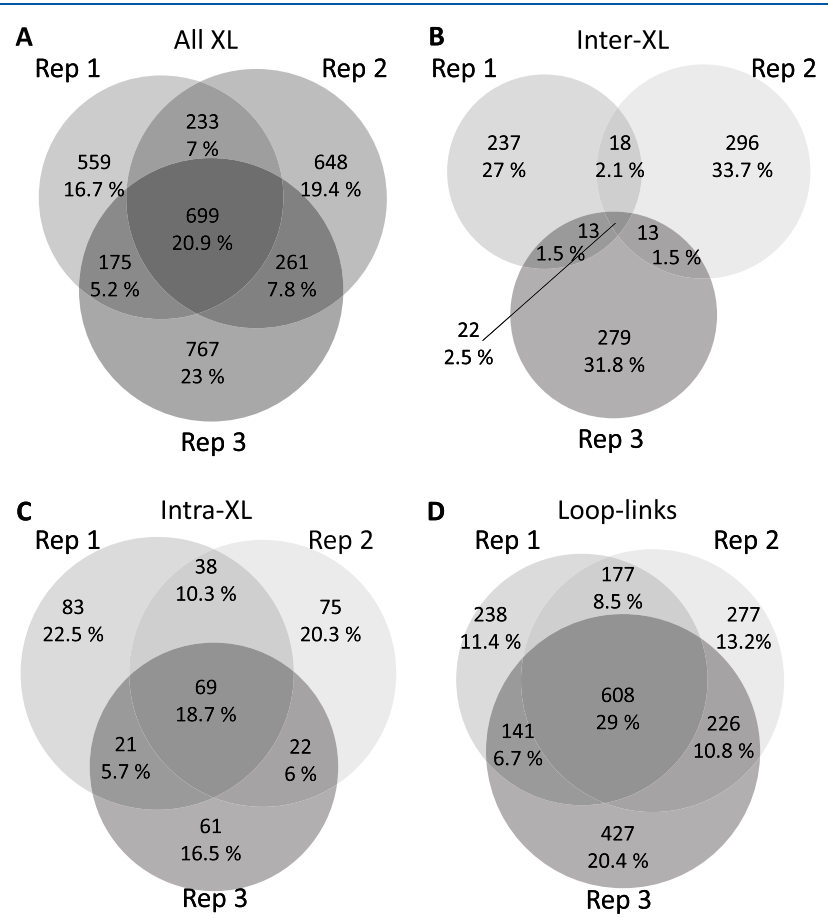

Figure 2. Venn diagram of the number of XLs in the three different replicates of N. meningitidis. XL experiments. (A) All XLs identified. (B) Inter-XLs. (C) Intra-XLs. (D) Loop-links. Areas and overlaps are proportional to the values indicated in each diagram, i.e., the number of identifications and percentage.

major reasons: they are easier to form, as they are not transient, and linear, thus easier to fragment evenly. We also identified 2 times more inter-XLs than intra-XLs, simply because they are a much larger group. Looking at the reproducibility, the LL data are within the expected range with $29 \%$ identified in all replicates (Figure 2). Increasing the number of replicates leads to a 45.2 and $25.6 \%$ increase (Tables S1A and S1B). Quite similar results were obtained for intra-XL (18.7\% overlap) with 45.2 and $19.5 \%$ increase per replicate. The results for the inter$\mathrm{XL}$ are different as a much lower overlap is observed between replicates. Indeed, $2.5 \%$ of inter-XLs are common to all replicates with 106.5 and $46.5 \%$ increase per replicate. We foresee several explanations for this result. One could be linked to the search since correct FDR assignments differ significantly when assigning inter- or intra-XL. Detecting significant amounts of true-positive inter-XLs in vivo is a general, unsolved problem of the whole field. Another explanation is the very low probability of the inter-XL event. It has been estimated that in a cell, each protein undergoes on average five interactions with another one. ${ }^{37,38}$ Considering that at least four inter-XLs could be detected per interaction, this would lead, for $N$. meningitidis, to an expected number of more than 40000 possible inter-XLs $(2000 \times 5 \times 4)$. Capturing only 300 inter-XLs per replicate among those 40000 possibilities leads statistically to a low overlap between two biological replicates. A low overlap is also observed for the nonlabeled peptides, with only 14 found in common in the three replicates for 190 identified in total (Figure S4). Another reason may stand in the 


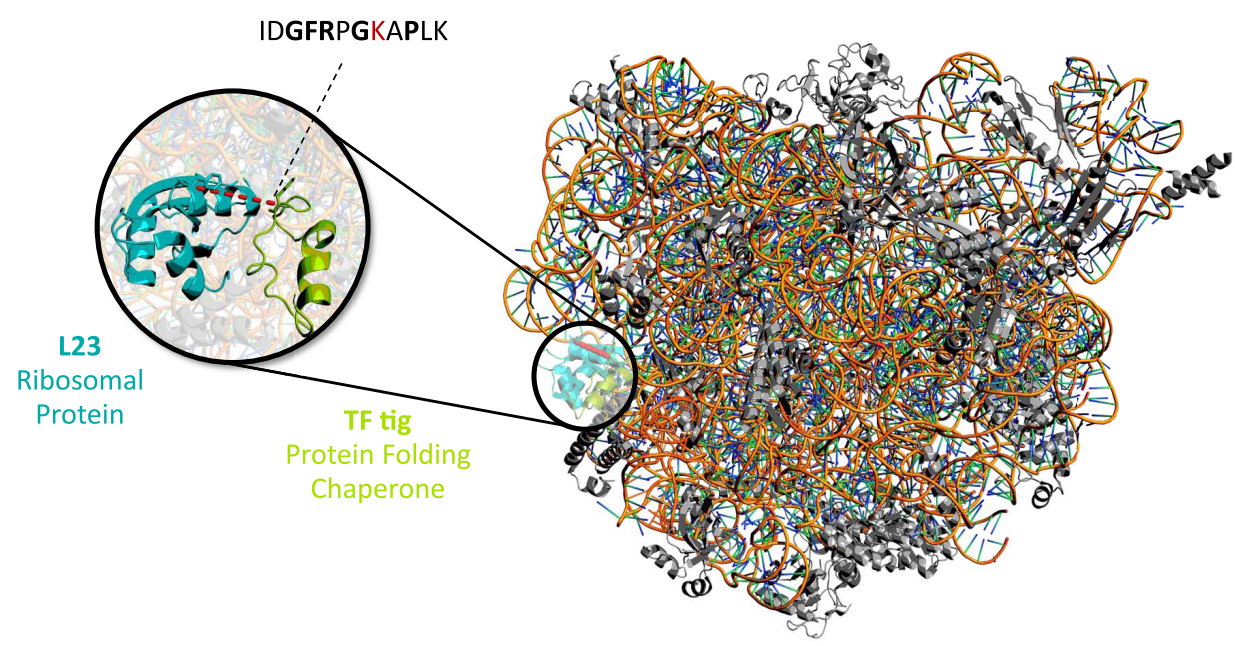

Figure 3. Ribbon structure of the E. coli ribosome $50 \mathrm{~S}$ subunit in complex with the TF tig (pdb $1 \mathrm{~W} 2 \mathrm{~B}) .{ }^{47}$ The left insert is a zoomed view of the cross-linked peptide (red dashes) between L23 (cyan) and TF tig (lime) proteins and its sequence.

fact that inter-XLs are much more transient than intra-XLs, which are often captured within a folded and structurally stable domain. Finally, the stochasticity of our DDA approach is also an unfavorable factor for the analysis of the complex mixture obtained in in vivo XL-MS experiments. A way to improve the situation would be to apply a match between run algorithm, but it is unfortunately not available so far in XL-MS software. A fractionation of the sample could also help to decrease its complexity and capture more inter-XLs.

Among all pairs of residues attached by NNP9, 685 were found to be between 2 lysines, 1874 between one $\mathrm{K}$ and one $\mathrm{S}$, $\mathrm{T}$ or $\mathrm{Y}$ and 783 between S, T or Y on either site (Table S3). In our experiment, the ratio of $\mathrm{K}$ and STY is roughly 50\%. This percentage is slightly higher than what is usually observed (33\%) ${ }^{39}$ which is probably due to the fact that NNP9 is more stable in water than the classical NHS ester, giving more time to find a (less) reactive site before being hydrolyzed. This statement is in agreement with the DE values where a ratio of $34.0 \%$ is found between K and STY labeled, respectively, 8477 and 4372 .

In addition to the cross-linked peptides, 4296 dead-ends were obtained representing the majority of all peptides (55\%, Table S5). The large number of dead-ends can be due to the fact that they are easy to form as they require only one site to react with the cross-linker. It could also be a bias in the identification since it is easier to produce comprehensive fragmentation from a linear peptide than from a branched one. The hydrolyzed dead-ends (DEs) are due to hydrolysis during the experiment and the dead-end quenched (DEQ) reacted with ammonia during the quenching step. The large number of DEQ identified, even after $3 \mathrm{~h}$ of labeling, shows the great stability of our cross-linker that includes NHS-carbamate groups. This enhanced stability ensures that our cross-linker could enter the cell and react with proteins before being hydrolyzed. For all dead-ends, increasing the number of replicates leads to an increase of 20 and $11 \%$, which is more in line with regular proteomics experiments and can be explained by the high abundance of these peptides.

Finally, only 83-108 nonlabeled peptides were identified per replicate. Compared to the 4972 peptides identified with a charge state of 3 or more in a full proteome analysis, this corresponds to a $98 \%$ removal of the nonlabeled peptides, showing the outstanding efficiency of our one-step enrichment.
The poor recovery between runs (14 peptides) is probably due, as for the cross-linked peptides, to the very low abundance of these peptides, combined with the fact that we sample a large ensemble, and the inherent stochasticity of our datadependent analysis (DDA) strategy.

Highlights on Selected Protein Assemblies. We then decided to mine the thousands of interactions captured in our in vivo experiments. We noticed that both soluble and membrane proteins arising from complexes covering each bacterial cell compartment, i.e., the cytoplasm, both the outer and inner membranes, and the intermembrane space with interaction with the peptidoglycan could be identified. To illustrate the important feature that our in vivo XL-MS approach indeed addresses the entire bacterial cell, we will further describe in detail three interesting protein assemblies: the ribosome present in the cytoplasm, the ATP synthase from the inner membrane, and the channeling of iron through the outer membrane porin PorB, which involves the peptidoglycan-binding protein $\mathrm{RmpM}$ and the periplasmic iron-binding protein $\mathrm{FbpA}$.

Starting with the cytoplasm, we analyzed the cross-links obtained on the 50 ribosomal proteins captured of the 56 present in the canonical ribosome (Table S4). Using the structure of the E. coli $70 \mathrm{~S}$ ribosome bound to the elongation factor G (PDB entry number 3JA1), we analyzed the distances between the cross-linked residues captured in our experiments. Among all cross-links identified in the triplicate, we were able to assign 561 distances on the ribosomal model. Almost all of the cross-links identified (97\%) were below $25 \AA$, which is the expected range of NNP9 (Figure S5). ${ }^{33}$ Among all of the distances measured in our triplicate, 98 XLs with 15 inter-XLs on nine different protein-protein interactions, 83 intra-XLs, and 255 loop-links involve directly 50 canonical ribosomal proteins. This result validates the stringency of our identification process and the quality of the distances captured during the in vivo labeling experiment with this trifunctional cross-linker.

Looking at the ribosomal interactome captured with our in vivo $\mathrm{XL}$ experiments, we found several interacting partners involved in the transcription-translation coupling. This is the case for the $\alpha$ subunit of RNA polymerase and two transcription factors: the transcription anti-termination protein NusA and the transcription-repair coupling factor mfd. These 


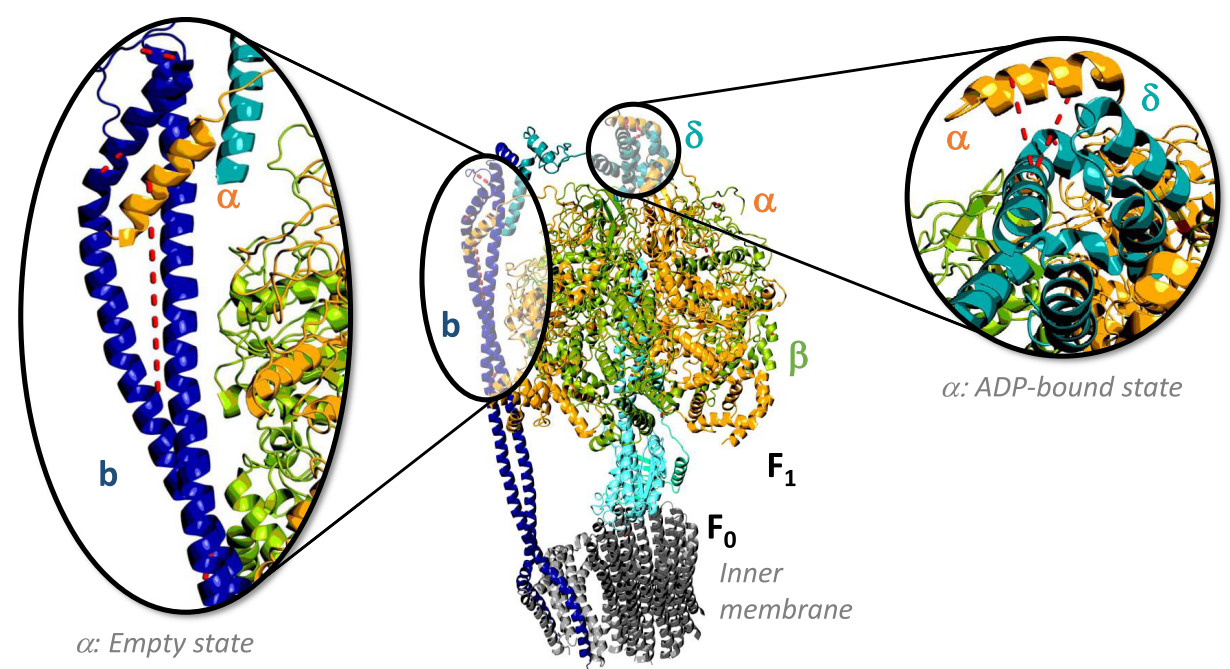

Figure 4. Ribbon structure of the E. coli ATP synthase F1/F0 with identified cross-links (red dashes). The left insert is a zoomed view of the crosslinked peptide between the $\alpha$ (gold) and $b$ (dark blue) subunits. The right insert is a zoomed view of the cross-linked peptide between the $\alpha$ (gold) and $\delta$ (turquoise) subunits.

two proteins regulate the speed of RNA polymerase. NusA has been shown to cause RNAP to pause, slowing down the overall rate of elongation. ${ }^{40}$ This pausing activity is important in regulating the level of transcript formation through attenuation, ${ }^{41,42}$ ensuring efficient termination at intrinsic transcription terminators, ${ }^{43-45}$ and in regulating the elongation rate of RNAP to ensure efficient coupling of transcription and translation. ${ }^{41}$ The transcription-repair coupling factor $\mathrm{mfd}$ is known to associate with RNAP and rescue stalled elongation transcription complexes. ${ }^{46}$ Altogether, these results confirm the close regulation of the transcription mechanism with a tight dialog between the RNAP, the ribosomal proteins and numerous regulatory factors. Continuing digging into the ribosomal interactome, we captured an interesting interaction between the trigger factor (TF) tig and the ribosomal protein L23, respectively, cross-linked via residues K48 (tig) and K25 (L23) (Figure 3).

The TF tig is the first protein-folding chaperone to interact with a nascent polypeptide chain as it emerges from the ribosome. $^{48,49}$ Remarkably, the TF tig cross-linked peptide IDGFRPGKAPLK contains the well-conserved TF motif GFRxGxxP responsible for the binding of the TF tig to L23 as captured by Ferbitz et al. in the $3 \mathrm{D}$ structure presented in Figure $3 .{ }^{47,50}$ Our ability to capture, in an unbiased large-scale method, specific protein motifs involved in protein-protein interactions shows the great power of our in vivo $\mathrm{XL}$ approach and its extreme sensitivity, required when dissecting relevant biological mechanisms.

The second complex we investigated is the ATP synthase F1/F0. Among the important protein complexes from the inner membrane, this one is well characterized with 23 unique cross-links (Figure 4) that involve subunits $\alpha, \beta, \delta, \varepsilon$, and $b$. Among the contacts, we characterized different interactions of the subunit $\alpha$ depending on its different states. In its empty conformation, the $\mathrm{N}$-terminal $\alpha$-helix of the $\alpha$ subunit interacts with the C-terminal region of the $b$ subunit as captured here by the cross-link shown in the left insert. On the contrary, in the ADP-bound state, the same N-terminus of the $\alpha$ subunit interacts with the $\delta$ subunit and gets cross-linked too (right insert, Figure 4). This highlights the high precision of our in vivo XL-MS approach and its ability to capture different conformational states of the same polypeptide chain. This means that our workflow is able to deliver structural information on functional machineries in their native state.

Finally, we investigated complexes from the intermembrane space and the outer membrane. This is a key region for pathogenic bacteria as it involves many proteins implicated in the virulence and the resistance to antibiotics. ${ }^{51}$ Moreover, many outer membrane proteins are also used in vaccines, as they are the ones first encountered by the immune system. We found that the peptidoglycan (PGN) binding protein RmpM interacts through its $\mathrm{N}$-terminal domain with the porin PorB. This interaction has already been described in the literature but without any precision. ${ }^{52,53}$ Here, porin lysines K75 and K103 of the $\beta$-sheets 3 and 4 of the transmembrane $\beta$-barrel were found to be cross-linked to the lysine K55 of RmpM (Figure S6), localizing very precisely the interaction. Just after the lysine K55 of RmpM, there is a specific stretch of residues composed of prolines and glutamic acids (PE), which could interact with the intermembrane edge of PorB rich in basic residues. A possibility is that the acidic PE stretch corresponds to a nondefined peptide of RmpG (a homologue of RmpM) modeled as a polyalanine peptide due to the lack of resolution observed in the PorB structure by Zeth et al. ${ }^{54} \mathrm{RmpM}$ also interacts with $\mathrm{FbpA}$, an intermembrane iron-binding protein, required for Ton-independent utilization of xenosiderophores for the iron carriage down to the inner membrane $\mathrm{FbpB} / \mathrm{C}$ ATP-dependent transporter. ${ }^{55}$ Note here that the presence of FbpA as the iron carrier is linked to the way N. meningitidis cells are cultured, on GBC medium supplemented with ferric ammonium citrate. In detail, residues K107 and K175 of FbpA were found to be cross-linked to K222 of RmpM. As shown in Figure S6, the cross-linked residues belong to the opposite faces of the (1) iron-binding motif for FbpA and (2) PGN recognition groove of the C-terminal domain of $\mathrm{RmpM}$, leaving accessible the main functions of both proteins. Altogether, this tripartite interaction suggests a tight channeling between the entry of iron in the bacteria through the porin PorB and its direct capture by $\mathrm{FbpA}$, both being hold close to each other by RmpM. In that case, RmpM would not only be a structural apparatus, which holds PGN and the OM together, but also an anchor for periplasmic binding proteins. 
This detailed picture was obtained on a complex involving membrane proteins, peptidoglycan-binding proteins, and iron transporters. It not only brought new information on the relationship between these proteins but also provided precise structural information that helps understanding the role, function, and interaction mechanisms between the partners in their native environment and in an unbiased way. These results confirm the requirement for more in vivo approaches and the power and sensitivity of our new in vivo XL-MS protocol.

\section{CONCLUSIONS}

We demonstrated here a new and straightforward approach to perform in vivo cross-linking and capture the interactome of an entire cell. It is based on the use of a trifunctional cross-linker (NNP9), which allows a very efficient one-step purification of cross-linked peptides and leads to high-quality MS/MS spectra that can be searched directly by an optimized search engine. We then successfully applied our new protocol on living bacterial cells of $N$. meningitidis. We identified more than 3300 cross-links in one triplicate analysis covering all of the compartments of the cell. With this approach, we identified more than 500 cross-links on the ribosome with $97 \%$ of them below the distance expected for our cross-linker. Among all of the contacts identified, we directly captured the critical binding motif of the trigger factor tig to the ribosome. Moreover, we were also able to pinpoint some structural details of the ATP synthase complex. Looking at important proteins involved in iron import and vaccines, we could structurally describe the channeling of iron where the intermembrane iron-binding protein $\mathrm{FbpA}$ is held closer to the porin PorB thanks to the peptidoglycan-binding protein RmpM. The precise binding site capture via our cross-linker allowed us to propose a new interaction mechanism between RmpM and PorB.

Overall, our new in vivo XL-MS protocol holds a great potential to capture new interactions between proteins in an entire cell in a nontargeted and unbiased large-scale manner. Moreover, it allows protein complexes to be probed in their functional state, which is of high importance to decipher relevant biological processes.

\section{ASSOCIATED CONTENT}

\section{SI Supporting Information}

The Supporting Information is available free of charge at https://pubs.acs.org/doi/10.1021/acs.analchem.0c04430.

Figure S1, chemical structure of NNP9; Figure S2, chemical reaction of the purification of labeled peptide via click chemistry; Figure S3, efficiency of the clickchemistry reaction on photocleavable beads; Figure S4, number of dead-ends and nonlabeled peptides identified across the three replicates, represented by Venn diagrams; Figure S5, distribution of cross-link distances of the ribosomal protein of N. meningitidis; and Figure S6, ribbon structure of RmpM in interaction with FbpA and the porin PorB (PDF)

Table S1, summary of the number of labeled proteins and cross-linked peptides identified in the in vivo XL-MS experiment; Table S2, summary of the number of proteins and peptides identified in a nonlabeled proteomic experiment; Table S3, summary of the amino acid labeled with NNP9; Table S4, list of all of the cross-linked peptides identified in the three replicates of N. meningitides; and Table S5, list of the dead-end peptides identified for the three replicates, with score, proteins, peptide sequences, and labeled sites (XLSX)

Cross-link peptide search parameters (TXT)

Protein database (TXT)

\section{AUTHOR INFORMATION}

\section{Corresponding Authors}

Martial Rey - Mass Spectrometry for Biology Unit, Department of Structural Biology and Chemistry, Institut Pasteur, CNRS USR 2000, 75015 Paris, France; ○ orcid.org/0000-0002-7378-1106; Email: martial.rey@ pasteur.fr

Julia Chamot-Rooke - Mass Spectrometry for Biology Unit, Department of Structural Biology and Chemistry, Institut Pasteur, CNRS USR 2000, 75015 Paris, France; ○ orcid.org/0000-0002-9427-543X; Email: julia.chamotrooke@pasteur.fr

\section{Authors}

Jonathan Dhenin - Mass Spectrometry for Biology Unit, Department of Structural Biology and Chemistry, Institut Pasteur, CNRS USR 2000, 75015 Paris, France

Youxin Kong - Pathogenesis of Vascular Infections, Department of Cell Biology and Infection, Institut Pasteur, INSERM U1225, 75015 Paris, France

Lucienne Nouchikian - Mass Spectrometry for Biology Unit, Department of Structural Biology and Chemistry, Institut Pasteur, CNRS USR 2000, 75015 Paris, France

Isaac Filella - Structural Bioinformatics Unit, Department of Structural Biology and Chemistry, Institut Pasteur, CNRS UMR 3528, 75015 Paris, France

Magalie Duchateau - Mass Spectrometry for Biology Unit, Department of Structural Biology and Chemistry, Institut Pasteur, CNRS USR 2000, 75015 Paris, France

Mathieu Dupré - Mass Spectrometry for Biology Unit, Department of Structural Biology and Chemistry, Institut Pasteur, CNRS USR 2000, 75015 Paris, France; (1) orcid.org/0000-0002-1845-0048

Riccardo Pellarin - Structural Bioinformatics Unit, Department of Structural Biology and Chemistry, Institut Pasteur, CNRS UMR 3528, 75015 Paris, France

Guillaume Duménil - Pathogenesis of Vascular Infections, Department of Cell Biology and Infection, Institut Pasteur, INSERM U1225, 75015 Paris, France

Complete contact information is available at: https://pubs.acs.org/10.1021/acs.analchem.0c04430

\section{Author Contributions}

The manuscript was written with contributions of all authors. All authors have given approval to the final version of the manuscript.

\section{Notes}

The authors declare no competing financial interest.

\section{ACKNOWLEDGMENTS}

All raw data, databases, and processing parameters are available on ProteomeXchange via PRIDE repository (data set identifier PXD021553). Synthesis and characterization of NNP9 were performed by the COBRA lab (Mont-Saint-Aignan, University of Rouen). This work has been supported by the Institut 
Pasteur, the CNRS, the Agence Nationale de la Recherche (CLICKMASSLINK-ANR 09-PIRI-0006, T4PNanoAction ANR-18-CE11-0022), the "Investissement d'Avenir" Bioinformatique program (grant BIP:BIP, ANR-10-BINF-03-13), and the EPIC-XS project number 823839 , funded by the Horizon 2020 programme of the European Union. The authors are grateful to $\mathrm{Q}$. Giai-Gianetto for the statistical analysis of crosslinked peptides.

\section{REFERENCES}

(1) Barysz, H. M.; Malmström, J. Mol. Cell. Proteomics 2018, 17, $1055-1066$.

(2) Liu, F.; Rijkers, D. T. S.; Post, H.; Heck, A. J. R. Nat. Methods 2015, 12, 1179-1184.

(3) Ryl, P. S. J.; Bohlke-Schneider, M.; Lenz, S.; Fischer, L.; Budzinski, L.; Stuiver, M.; Mendes, M. M. L.; Sinn, L.; O’Reilly, F. J.; Rappsilber, J. J. Proteome Res. 2020, 19, 327-336.

(4) de Jong, L.; de Koning, E. A.; Roseboom, W.; Buncherd, H.; Wanner, M. J.; Dapic, I.; Jansen, P. J.; van Maarseveen, J. H.; Corthals, G. L.; Lewis, P. J.; Hamoen, L. W.; de Koster, C. G. J. Proteome Res. 2017, 16, 2457-2471.

(5) Kaake, R. M.; Wang, X.; Burke, A.; Yu, C.; Kandur, W.; Yang, Y.; Novtisky, E. J.; Second, T.; Duan, J.; Kao, A.; Guan, S.; Vellucci, D.; Rychnovsky, S. D.; Huang, L. Mol. Cell. Proteomics 2014, 13, 35333543.

(6) Matzinger, M.; Mechtler, K. J. Proteome Res. 2021, 20, 78-93.

(7) Rinner, O.; Seebacher, J.; Walzthoeni, T.; Mueller, L. N.; Beck, M.; Schmidt, A.; Mueller, M.; Aebersold, R. Nat. Methods 2008, 5, 315.

(8) Chen, Z. A.; Jawhari, A.; Fischer, L.; Buchen, C.; Tahir, S.; Kamenski, T.; Rasmussen, M.; Lariviere, L.; Bukowski-Wills, J.-C.; Nilges, M.; Cramer, P.; Rappsilber, J. EMBO J. 2010, 29, 717-726.

(9) Lauber, M. A.; Reilly, J. P. Anal. Chem. 2010, 82, 7736-7743.

(10) Tinnefeld, V.; Venne, A. S.; Sickmann, A.; Zahedi, R. P. J. Proteome Res. 2017, 16, 459-469.

(11) Schmidt, R.; Sinz, A. Anal. Bioanal. Chem. 2017, 409, 23932400.

(12) Leitner, A.; Reischl, R.; Walzthoeni, T.; Herzog, F.; Bohn, S.; Förster, F.; Aebersold, R. Mol. Cell. Proteomics 2012, 11, No. M111.014126.

(13) Leitner, A.; Joachimiak, L. A.; Unverdorben, P.; Walzthoeni, T.; Frydman, J.; Förster, F.; Aebersold, R. Proc. Natl. Acad. Sci. U.S.A. 2014, 111, 9455-9460.

(14) Kelstrup, C. D.; Bekker-Jensen, D. B.; Arrey, T. N.; Hogrebe, A.; Harder, A.; Olsen, J. V. J. Proteome Res. 2018, 17, 727-738.

(15) Ishmael, F. T.; Alley, S. C.; Benkovic, S. J. J. Biol. Chem. 2002, 277, 20555-20562.

(16) Tang, X.; Bruce, J. E. Mol. BioSyst. 2010, 6, 939-947.

(17) Chowdhury, S. M.; Du, X.; Tolić, N.; Wu, S.; Moore, R. J.; Mayer, M. U.; Smith, R. D.; Adkins, J. N. Anal. Chem. 2009, 81, $5524-5532$.

(18) Sohn, C. H.; Agnew, H. D.; Lee, J. E.; Sweredoski, M. J.; Graham, R. L. J.; Smith, G. T.; Hess, S.; Czerwieniec, G.; Loo, J. A.; Heath, J. R.; Deshaies, R. J.; Beauchamp, J. L. Anal. Chem. 2012, 84, 2662-2669.

(19) Rey, M.; Dupré, M.; Lopez-Neira, I.; Duchateau, M.; ChamotRooke, J. Anal. Chem. 2018, 90, 10707-10714.

(20) Collins, C. J.; Schilling, B.; Young, M.; Dollinger, G.; Guy, R. K. Bioorg. Med. Chem. Lett. 2003, 13, 4023-4026.

(21) Petrotchenko, E. V.; Serpa, J. J.; Borchers, C. H. Mol. Cell. Proteomics 2011, 10, No. M110.001420.

(22) Fischer, L.; Chen, Z. A.; Rappsilber, J. J. Proteomics 2013, 88, $120-128$.

(23) Petrotchenko, E. V.; Borchers, C. H. BMC Bioinf. 2010, 11, 64. (24) Götze, M.; Pettelkau, J.; Schaks, S.; Bosse, K.; Ihling, C. H.; Krauth, F.; Fritzsche, R.; Kühn, U.; Sinz, A. J. Am. Soc. Mass Spectrom. 2012, 23, 76-87.
(25) Yang, B.; Wu, Y.-J.; Zhu, M.; Fan, S.-B.; Lin, J.; Zhang, K.; Li, S.; Chi, H.; Li, Y.-X.; Chen, H.-F.; Luo, S.-K.; Ding, Y.-H.; Wang, L.H.; Hao, Z.; Xiu, L.-Y.; Chen, S.; Ye, K.; He, S.-M.; Dong, M.-Q. Nat. Methods 2012, 9, 904.

(26) Rasmussen, M. I.; Refsgaard, J. C.; Peng, L.; Houen, G.; Høirup, P. J. Proteomics 2011, 74, 1871-1883.

(27) Lu, L.; Millikin, R. J.; Solntsev, S. K.; Rolfs, Z.; Scalf, M.; Shortreed, M. R.; Smith, L. M. J. Proteome Res. 2018, 17, 2370-2376.

(28) Sarpe, V.; Rafiei, A.; Hepburn, M.; Ostan, N.; Schryvers, A. B.; Schriemer, D. C. Mol. Cell. Proteomics 2016, 15, 3071-3080.

(29) Kao, A.; Chiu, C.; Vellucci, D.; Yang, Y.; Patel, V. R.; Guan, S.; Randall, A.; Baldi, P.; Rychnovsky, S. D.; Huang, L. Mol. Cell. Proteomics 2011, 10, No. M110.002212.

(30) Müller, M. Q.; Dreiocker, F.; Ihling, C. H.; Schäfer, M.; Sinz, A. Anal. Chem. 2010, 82, 6958-6968.

(31) Sinz, A. Anal. Bioanal. Chem. 2017, 409, 33-44.

(32) Liu, F.; Lössl, P.; Scheltema, R.; Viner, R.; Heck, A. J. R. Nat. Commun. 2017, 8, No. 15473.

(33) Nury, C.; Redeker, V.; Dautrey, S.; Romieu, A.; van der Rest, G.; Renard, P.-Y.; Melki, R.; Chamot-Rooke, J. Anal. Chem. 2015, 87, $1853-1860$

(34) Guilhen, C.; Taha, M.-K.; Veyrier, F. J. Front. Cell. Infect. Microbiol. 2013, 3, 102.

(35) Dupré, M.; Duchateau, M.; Malosse, C.; Borges-Lima, D.; Calvaresi, V.; Podglajen, I.; Clermont, D.; Rey, M.; Chamot-Rooke, J. J. Proteome Res. 2020, 202-211.

(36) Tornøe, C. W.; Christensen, C.; Meldal, M. J. Org. Chem. 2002, 67, 3057-3064.

(37) Mishra, N. C.Introduction to Proteomics: Principles and Applications; Wiley, 2010.

(38) Grigoriev, A. Nucleic Acids Res. 2003, 31, 4157-4161.

(39) Iacobucci, C.; Piotrowski, C.; Aebersold, R.; Amaral, B. C.; Andrews, P.; Bernfur, K.; Borchers, C.; Brodie, N. I.; Bruce, J. E.; Cao, Y.; Chaignepain, S.; Chavez, J. D.; Claverol, S.; Cox, J.; Davis, T.; Degliesposti, G.; Dong, M.-Q.; Edinger, N.; Emanuelsson, C.; Gay, M.; Götze, M.; Gomes-Neto, F.; Gozzo, F. C.; Gutierrez, C.; Haupt, C.; Heck, A. J. R.; Herzog, F.; Huang, L.; Hoopmann, M. R.; Kalisman, N.; Klykov, O.; Kukačka, Z.; Liu, F.; MacCoss, M. J.; Mechtler, K.; Mesika, R.; Moritz, R. L.; Nagaraj, N.; Nesati, V.; Neves-Ferreira, A. G. C.; Ninnis, R.; Novák, P.; O’Reilly, F. J.; Pelzing, M.; Petrotchenko, E.; Piersimoni, L.; Plasencia, M.; Pukala, T.; Rand, K. D.; Rappsilber, J.; Reichmann, D.; Sailer, C.; Sarnowski, C. P.; Scheltema, R. A.; Schmidt, C.; Schriemer, D. C.; Shi, Y.; Skehel, J. M.; Slavin, M.; Sobott, F.; Solis-Mezarino, V.; Stephanowitz, H.; Stengel, F.; Stieger, C. E.; Trabjerg, E.; Trnka, M.; Vilaseca, M.; Viner, R.; Xiang, Y.; Yilmaz, S.; Zelter, A.; Ziemianowicz, D.; Leitner, A.; Sinz, A. Anal. Chem. 2019, 91, 6953-6961.

(40) Richardson, J. P.; Greenblatt, J. Control of RNA Chain Elongation and Termination. In Escherichia coli and Salmonella: Cellular and Molecular Biology; Neidhardt, F. C., Ed.; American Society for Microbiology Press: Washington, DC, 1996; pp 822-884.

(41) Landick, R.; Roberts, J. W. Science 1996, 273, 202-203.

(42) Yakhnin, A. V.; Babitzke, P. Proc. Natl. Acad. Sci. U.S.A. 2002, 99, 11067-11072.

(43) von Hippel, P. H. Science 1998, 281, 660-665.

(44) Ingham, C. J.; Dennis, J.; Furneaux, P. A. Mol. Microbiol. 1999, $31,651-663$.

(45) Gusarov, I.; Nudler, E. Cell 2001, 107, 437-449.

(46) Ho, H. N.; van Oijen, A. M.; Ghodke, H. Nat. Commun. 2018, 9, No. 1570.

(47) Ferbitz, L.; Maier, T.; Patzelt, H.; Bukau, B.; Deuerling, E.; Ban, N. Nature 2004, 431, 590-596.

(48) Wegrzyn, R. D.; Deuerling, E. Cell. Mol. Life Sci. 2005, 62, 2727-2738.

(49) Craig, E. A.; Eisenman, H. C.; Hundley, H. A. Curr. Opin. Microbiol. 2003, 6, 157-162.

(50) Kramer, G.; Rauch, T.; Rist, W.; Vorderwülbecke, S.; Patzelt, H.; Schulze-Specking, A.; Ban, N.; Deuerling, E.; Bukau, B. Nature 2002, 419, 171-174. 
(51) Choi, U.; Lee, C.-R. Front. Microbiol. 2019, 10, No. 953.

(52) Maharjan, S.; Saleem, M.; Feavers, I. M.; Wheeler, J. X.; Care, R.; Derrick, J. P. Microbiology 2016, 162, 364-375.

(53) Grizot, S.; Buchanan, S. K. Mol. Microbiol. 2004, 51, 10271037.

(54) Zeth, K.; Kozjak-Pavlovic, V.; Faulstich, M.; Fraunholz, M.; Hurwitz, R.; Kepp, O.; Rudel, T. Biochem. J. 2013, 449, 631-642.

(55) Strange, H. R; Zola, T. A.; Cornelissen, C. N. Infect. Immun. 2011, 79, 267-278. 